\title{
Villoglandular adenocarcinoma of the uterine cervix: a systematic review and meta-analysis
}

\author{
Anna K. Dietl ${ }^{1}$ (1) $\cdot$ Matthias W. Beckmann ${ }^{1} \cdot$ Konrad Aumann ${ }^{2,3}$
}

Received: 8 February 2021 / Accepted: 17 April 2021 / Published online: 25 May 2021

(c) The Author(s) 2021

\begin{abstract}
Purpose Villoglandular adenocarcinoma (VGA) of the uterine cervix has been classified as a rare subtype of cervical adenocarcinoma with good prognosis. A conservative surgical approach is considered feasible. The main risk factor is the presence of other histologic types of cancer.

In this largest systematic review to date, we assess oncological outcomes associated with conservative therapy compared to those associated with invasive management in the treatment of stage Ia and $\mathrm{Ib}_{1}$ VGA.

Methods Case series and case reports identified by searching the PubMed database were eligible for inclusion in this review (stage $\mathrm{Ia}-\mathrm{Ib}_{1}$ ).

Results A total of 271 patients were included in our literature review. 54 (20\%) patients were treated by "conservative management" (conization, simple hysterectomy, and trachelectomy) and 217 (80\%) by "invasive management" (radical hysterectomy \pm radiation, hysterectomy, and radiation). Recurrences of disease (RODs) were found in the conservative group in two (4\%) cases and in the invasive group in nine (4\%) cases. There was no significant difference in disease-free survival (DFS) according to conservative or invasive treatment $(p=0.75)$. The histology of VGA may be complex with underlying usual adenocarcinoma (UAC) combined with VGA.

Conclusion The excellent prognosis of pure VGA and the young age of the patients may justify the management of this tumor using a less radical procedure. The histological diagnosis of VGA is a challenge, and pretreatment should not be based solely on a simple punch biopsy but rather a conization with wide tumor-free margins.
\end{abstract}

Keywords Villoglandular adenocarcinoma $\cdot$ Cervix $\cdot$ Conservative therapy $\cdot$ Invasive therapy $\cdot$ Review

\section{Introduction}

Adenocarcinoma of the cervix comprises for $15-20 \%$ of all carcinomas of the uterine cervix. There is evidence that the absolute incidence of adenocarcinoma is increasing, especially in women younger than 35 years $[1,2]$.

In 1989, Young and Scully [3] drew attention to a rare subtype of cervical adenocarcinoma, the villoglandular adenocarcinoma (VGA). The International Endocervical

Anna K. Dietl

anna.dietl@uk-erlangen.de

1 Department of Obstetrics and Gynecology, University of Erlangen, 91054 Erlangen, Germany

2 Center for Pathology Allgaeu, Klinikverbund Allgaeu, Kempten, Germany

3 Faculty of Medicine, University of Freiburg, 79106 Freiburg, Germany
Adenocarcinoma Criteria and Classification (IECC) declared that VGA is a human papillomavirus (HPV) - associated adenocarcinoma [4]. The incidence of this subtype is reported as $4-9 \%$ of usual cervical adenocarcinoma (UAC) $[5,6]$.

The standard surgery for patients with stage $\mathrm{Ia}_{2}-\mathrm{Ib}_{1}$ cervical cancer is radical hysterectomy (RH) and lymphadenectomy (LNE). However, this procedure does not preserve fertility and can significantly affect quality of life.

The majority of reports revealed that the long-term prognosis of VGA is more favorable than UAC. Non-radical surgery or ovarian preservation might be safe for patients with pure early-stage VGA.

The aim, on the one hand, should be to avoid overtreatment by determining an exact diagnosis to preserve the fertility of young women and, on the other hand, to identify risk factors and offer optimal therapy for the VGA-tumor. Thus, the choice of treatment in patients with VGA remains 
controversial, and clarity is needed. In this largest systematic review to date, we assess oncological outcomes associated with conservative therapy compared to those associated with invasive management in the treatment of stage $\mathrm{Ia}$ and $\mathrm{Ib}_{1}$ VGA.

\section{Materials and methods}

This systematic review was based on the PRISMA guidelines [7] (Fig. 1). Published reports were identified by searches of PubMed and from references of relevant articles published from 1989 (the year VGA was described by Young and Scully [3]) to 2021. We used the search terms "villoglandular adenocarcinoma of the uterine cervix", "early-stage cervical cancer", "cone biopsy", and "radical hysterectomy". All papers that reported VGA in the abstracts and contained adequate information (including patient age, stage, primary treatment and postoperative treatment (radiotherapy and chemotherapy), clinical course, and followup) were included. The review included only women with "early-stage cervical cancer" and excluded patients with stages $\mathrm{Ib}_{2}-\mathrm{IIIb}$. Tumors were included if they were in stage I not otherwise specified (nos) when a study was published before the subdivision into $\mathrm{Ib}_{1}$ and $\mathrm{Ib}_{2}$ was implemented and

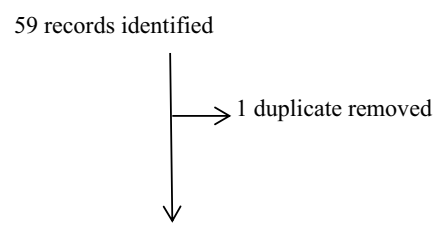

58 records screened

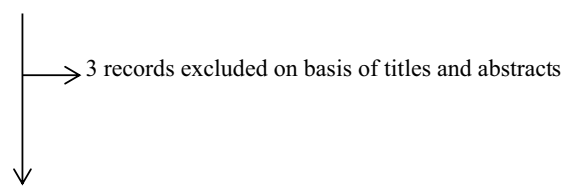

55 full-text articles assessed

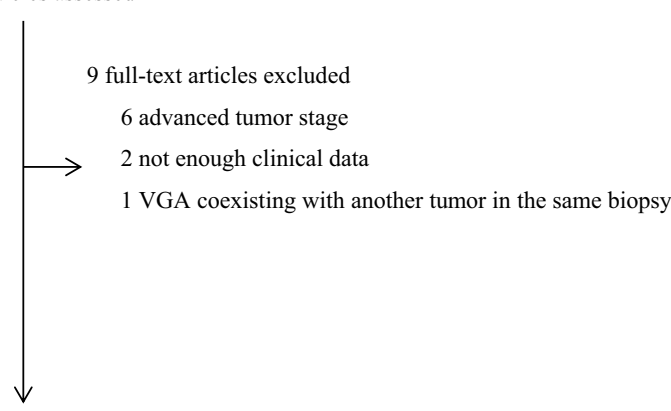

46 papers included (271 patients)

Fig. 1 Search strategy and exclusion criteria (adapted from PRISMA [7]) if the tumor would be classified as stage $\mathrm{Ib}_{1}$ according to a relevant clinical and pathological description.

If the preoperative diagnosis was made only by a single biopsy (punch biopsy), then the final diagnosis with the surgical specimen (cervix, uterus) did not always confirm the initial diagnosis due to the fact that small biopsies often contain tissue from the surface of the exophytic tumor only. For a proper diagnosis of VGA histological evaluation of tumor including its basis is mandatory. In 14 cases, the diagnosis of VGA was made primarily by single biopsy, and the surgical specimen resulted in $n=5 \mathrm{VGA}+\mathrm{UAC}, n=3 \mathrm{VGA}+$ squamous cell carcinoma, $n=5 \mathrm{UAC}$, and $n=1$ endometrial adenocarcinoma [8-17]. These cases were excluded from the study.

To compare disease-free survival (DFS) distributions between conservative and invasive treatment groups, a meta-analysis including a total of 44 papers and a total of 232 patients was carried out. Whenever individual follow-up data were not available, they were estimated by equidistantly dividing the respective time intervals. Statistics were calculated using SPSS Version 25. Data analysis was performed with descriptive statistics and Kaplan-Meier curves. DFS outcomes were compared with the log rank test.

\section{Results}

The PubMed search generated 59 reports and comprised a total of 398 patients. Of these, 271 patients met the inclusion criteria and underwent conservative management $(n=54$ : conization, simple hysterectomy, trachelectomy, without adjuvant therapy) or invasive management ( $n=217$ : radical hysterectomy (RH) with or without adjuvant therapy, hysterectomy with adjuvant therapy). There was no significant difference in DFS according to conservative or invasive treatment (Fig. 2, log rank, $p=0.75$ ).

\section{Conservative management}

We found 21 reports (stage $\mathrm{Ia}_{1}, \mathrm{Ib}_{1}$, I nos) describing conization in 28 patients, hysterectomy in 21 patients, and trachelectomy in five patients. Nine patients underwent pelvic lymph node dissection, lymph node biopsy or lymph node sampling (Table 1).

Negative LVI (lymphovascular invasion) status was reported in 32 patients, positive LVI status was reported in no patients, and LVI status was not reported in 22 patients.

Two patients (4\%) had recurrent disease: one in the cervix 25 months after conization [5]. The reported margins of the conization were uninvolved but were close to the tumor. She underwent RH and was alive after 62 months of follow-up. The second patient had a cone biopsy (VGA-tumor, $2.4 \mathrm{~mm}$ depth invasion, all resection margins clear) [8]. Close 


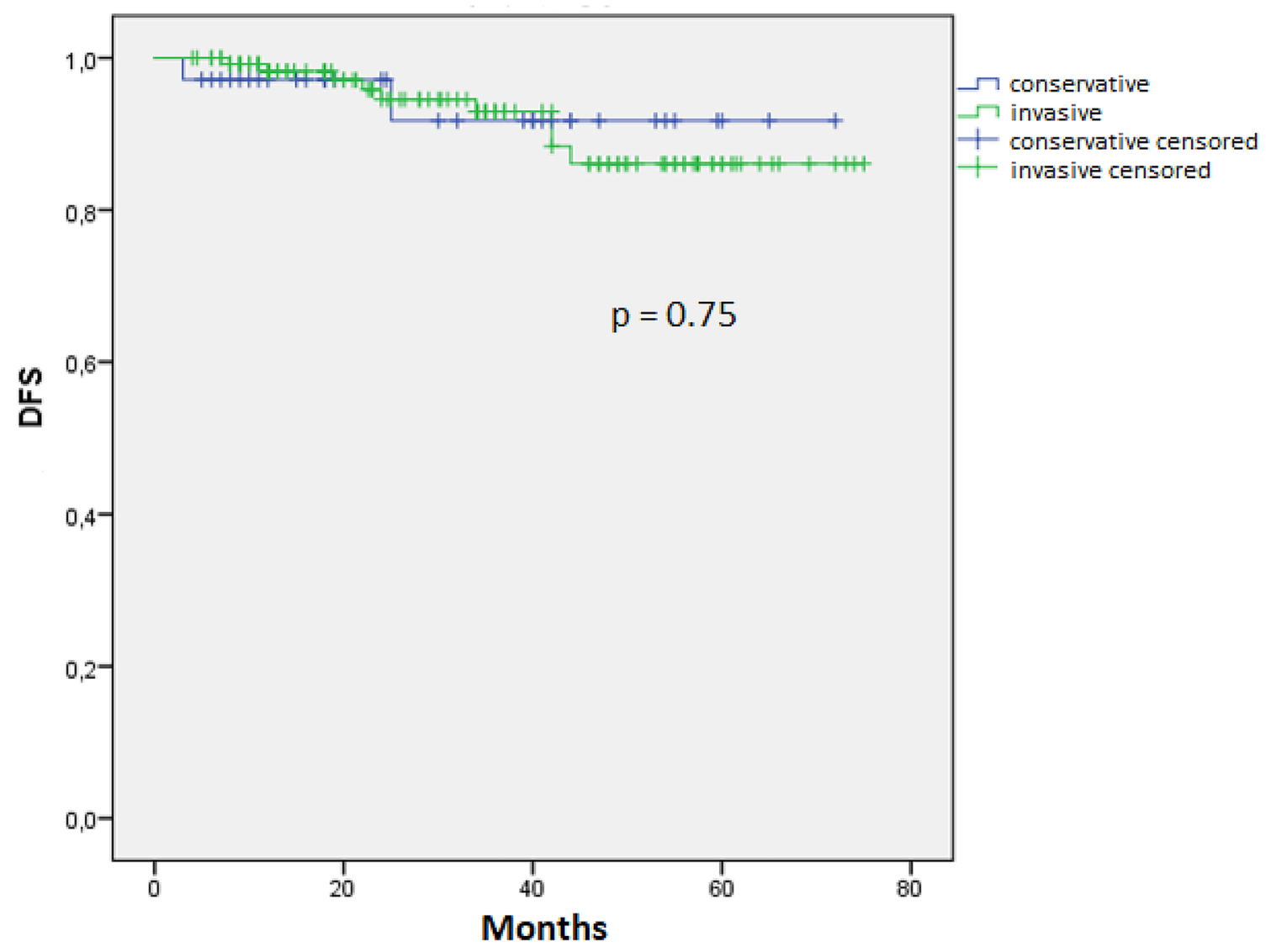

Fig. 2 DFS of VGA by conservative and invasive treatment

follow-up was recommended due to the histology. 3 months later, a cervical recurrence was noted. A biopsy showed a continuum from a well-differentiated adenocarcinoma with a villoglandular pattern to a poorly differentiated carcinoma. Rapid tumor progression followed chemoradiation therapy, and the patient died due to complications of an extensive pelvic tumor. Histology was sent for external review and was classified as a well-differentiated adenocarcinoma with a marked villoglandular pattern.

Seven pregnancies were reported in the "conservative management" group. In two patients, successful pregnancies were achieved following conization at the 14th/16th week of gestation $[18,19]$. Four patients delivered $1-5$ years after the conization $[9,10,20,21]$. One patient received a punch biopsy and conization during pregnancy and later underwent a trachelectomy and lymphadenectomy (LNE) of the tumor during cesarean section [22].

\section{Invasive management}

We found reports of 217 patients with tumor stages $\mathrm{Ia}_{1}, \mathrm{Ia}_{2}$, $\mathrm{Ib}_{1}$, and I nos (Table 2). Recurrent disease was seen in nine (5\%) patients, and three deaths were reported.
Among the nine patients with recurrence, one patient with FIGO stage $\mathrm{Ib}_{1}$ received a nerve-sparing laparoscopic RH, bilateral salpingo-oophorectomy, and pelvic LNE [6]. The histology showed well-differentiated VGA, and the infiltration depth was $5 \mathrm{~mm}$. The tumor recurred in the pelvic cavity after 8 months. At explorative laparotomy, the pelvic tumor was removed, and the histology revealed a UAC.

Another patient showed a VGA of the cervix after an uncomplicated vaginal delivery, and an RH with LNE was performed [23]. 44 months thereafter, the VGA recurred in the episiotomy scar.

One patient in the study by Korach et al. [10] was initially misdiagnosed with VGA instead of cervical adenocarcinoma. The tumor recurred 2 years after $\mathrm{RH}$, and the patient died a few months later.

The case series of Ju et al. [24] reported four metastases after RH, two in the ovaries, one in the liver and one on the vaginal vault. One patient had progressive disease after bilateral salpingo-oophorectomy because of ovarian metastasis and died 30 months later. The three patients with intraabdominal metastasis all underwent laparoscopic RH. 
Table 1 Literature review of conservative management for VGA

\begin{tabular}{|c|c|c|c|c|c|}
\hline & $\begin{array}{l}\text { Num- } \\
\text { ber of } \\
\text { patients }\end{array}$ & $\begin{array}{l}\text { Average age, } \\
\text { years (range) }\end{array}$ & FIGO stage & Surgery & Outcome (follow-up, months) \\
\hline Young and Scully 1989 [3] & 6 & $33(23-54)^{\mathrm{a}}$ & I nos & $\begin{array}{l}1 \mathrm{CON} \\
5 \mathrm{SH}\end{array}$ & $\operatorname{NED}(24-168)^{\mathrm{a}}$ \\
\hline Jones et al. 1993 [32] & 5 & $37(27-54)^{\mathrm{a}}$ & I nos & $\mathrm{CON}$ & NED (13-55) \\
\hline $\begin{array}{l}\text { Skopelitou and Hadjiyannakis } \\
1996 \text { [44] }\end{array}$ & 1 & 21 & $\mathrm{Ib}_{1}$ & $\mathrm{CON}$ & NED (12) \\
\hline Novotny and Ferlisi 1997 [45] & 3 & $35(25-48)$ & I nos & $\begin{array}{l}2 \mathrm{CON} \\
1 \mathrm{SH}\end{array}$ & NED (9-32) \\
\hline Borgo et al. 1998 [46] & 1 & 26 & $\mathrm{Ib}_{1}$ & $\mathrm{CON}$ & NED (40) \\
\hline Bouman et al. 1999 [9] & 1 & 26 & $\mathrm{Ib}_{1}$ & $\mathrm{CON}$ & $\begin{array}{l}\text { NED (15) delivery } 15 \text { months after } \\
\text { CON at } 36 \text { weeks }\end{array}$ \\
\hline Chang et al. 1999[47] & 2 & $40(35-44)$ & I nos & SH & NED (8-11) \\
\hline Hoffman et al. 2001 [20] & 1 & 28 & $\mathrm{Ib}_{1}$ & $\begin{array}{l}\text { CON (amputation of the cervical } \\
\text { portio) }\end{array}$ & NED (40) delivery at 36 weeks \\
\hline Falcon et al. 2006 [21] & 1 & 34 & $\mathrm{Ib}_{1}$ & $\mathrm{CON}$ & $\begin{array}{l}\text { NED (96) delivery } 60 \text { months } \\
\text { after CON }\end{array}$ \\
\hline Macdonald et al. 2006 [8] & 1 & 32 & $\mathrm{Ib}_{1}$ & $\mathrm{CON}$ & $\begin{array}{l}\text { ROD } 3 \text { months after CON recur- } \\
\text { rence (cervix), underwent RAD/ } \\
\text { CT, DOD (tumor progression, } \\
\text { UAC, second opinion) }\end{array}$ \\
\hline Lavie et al. 2008[18] & 1 & 31 & $\mathrm{Ib}_{1}$ & $\begin{array}{l}\text { CON (14th week of gestation) } \\
\text { CRH ( } 37 \text { th week) }\end{array}$ & NED (18) \\
\hline Korach et al. 2009[10] & 5 & $42(33-65)$ & $\begin{array}{l}2 \mathrm{Ib}_{1} \\
3 \mathrm{Ia}_{1}\end{array}$ & $\begin{array}{l}2 \mathrm{CON} \\
2 \mathrm{SH} \\
1 \mathrm{SH}+\mathrm{BSO}+\mathrm{LN} \text { sampling }\end{array}$ & NED (72-120) 1 term delivery \\
\hline Takai et al. 2010 [19] & 1 & 28 & $\mathrm{Ib}_{1}$ & CON (16 weeks of gestation) & NED (44) delivery at 38 weeks \\
\hline Hagiwara et al. 2013 [28] & 1 & 34 & $\mathrm{Ib}_{1}$ & SH + LN-biopsy & NED (154) \\
\hline Lataifeh et al. 2013 [22] & 3 & $30(27-32)$ & $\mathrm{Ib}_{1}$ & $\begin{array}{l}1 \mathrm{CS} \text { and CON, trachelectomy } \\
\text { and LNE } \\
2 \text { trachelectomy and LNE }\end{array}$ & NED (6-60) \\
\hline Kim et al. 2014 [5] & 5 & $37(32-44)$ & $\begin{array}{l}3 \mathrm{Ia}_{1} \\
2 \mathrm{Ib}_{1}\end{array}$ & $\begin{array}{l}4 \mathrm{CON} \\
1 \mathrm{LAVH}+\mathrm{LNE}\left(\mathrm{Ia}_{1}\right)\end{array}$ & $\begin{array}{l}4 \text { NED (18-55) } 1 \text { ROD } 25 \text { months } \\
\text { after CON recurrence (cervix), } \\
\text { underwent RH, NED (62) }\end{array}$ \\
\hline Dilley et al. 2015 [48] & 2 & $35(33-37)$ & $\mathrm{Ib}_{1}$ & $\begin{array}{l}1 \mathrm{CON} \\
1 \mathrm{RoHE}\end{array}$ & NED (18-41) \\
\hline Guo et al. 2018 [6] & 3 & $32(28-35)$ & $\mathrm{Ib}_{1}$ & $\begin{array}{l}2 \mathrm{CON} \\
1 \text { vag. trachelectomy }+\mathrm{LNE}\end{array}$ & NED (5-19) \\
\hline Ju et al. 2018 [24] & 3 & $43(28-56)$ & $\begin{array}{l}1 \mathrm{Ib}_{1} \\
2 \mathrm{Ia}_{1}\end{array}$ & $\begin{array}{l}1 \mathrm{CON} \\
1 \mathrm{VH}+\mathrm{BSO} \\
1 \mathrm{TLH}\end{array}$ & NED (44-65) \\
\hline Wei et al. 2018 [17] & 4 & $37(24-55)$ & $\mathrm{Ib}_{1}$ & $\begin{array}{l}2 \mathrm{CON} \\
1 \mathrm{TLH}+\mathrm{BSO} \\
1 \mathrm{TLH}+\mathrm{BSO}+\mathrm{LNE}\end{array}$ & NED (22-38) \\
\hline Chen et al 2021 [29] & 4 & $45(38-52)$ & $\begin{array}{l}3 \mathrm{Ib}_{1} \\
1 \mathrm{Ia}_{2}\end{array}$ & $\begin{array}{l}1 \mathrm{SH}+\mathrm{BS} \\
1 \mathrm{SH}+\mathrm{BSO} \\
1 \mathrm{TLH}+\mathrm{BS} \\
1 \text { trachelectomy + LNE }\end{array}$ & NED (25-90) \\
\hline
\end{tabular}

CON conization, $C S$ cesarean section, $C R H$ cesarean radical hysterectomy, $L A V H$ laparoscopic-assisted vaginal hysterectomy, $S H$ simple hysterectomy, $V H$ vaginal hysterectomy, $T L H$ total laparoscopic hysterectomy, $R O H E$ robot-assisted hysterectomy, $B S O$ bilateral salpingo-oophorectomy, $B S$ bilateral salpingectomy, $R A D$ radiation, $C T$ chemotherapy, nos not otherwise specified, $U A C$ usual adenocarcinoma. $L N E$ lymphadenectomy, $D O D$ dead of disease, $N E D$ no evidence of disease, $R O D$ recurrence of disease

${ }^{a}$ Including all patients of both groups 
Table 2 Literature review of invasive management for VGA

\begin{tabular}{|c|c|c|c|c|c|}
\hline & $\begin{array}{l}\text { Num- } \\
\text { ber of } \\
\text { patients }\end{array}$ & $\begin{array}{l}\text { Average Age, } \\
\text { year (range) }\end{array}$ & FIGO stage & $\begin{array}{l}\text { Surgery and/or additional treat- } \\
\text { ment }\end{array}$ & Outcome (follow-up, months) \\
\hline Young and Scully 1989 [3] & 7 & $33(23-54)^{\mathrm{a}}$ & I nos & $\begin{array}{l}4 \mathrm{RH}+\mathrm{LNE} \\
3 \mathrm{RH}\end{array}$ & $\operatorname{NED}(24-168)^{\mathrm{a}}$ \\
\hline Hopson et al. 1990 [13] & 3 & $36(28-42)$ & $\mathrm{Ib}$ & $3 \mathrm{RH}+\mathrm{LNE}$ & $\begin{array}{l}\text { NED (1 uneventful hospital } \\
\text { course, } 2: 8 \mathrm{mths})\end{array}$ \\
\hline Jones et al. 1993 [32] & 19 & $37(27-54)^{\mathrm{a}}$ & I nos & $\begin{array}{l}4 \mathrm{SH}+\mathrm{RAD} \\
15 \mathrm{RH}\end{array}$ & $\operatorname{NED}(7-77)^{\mathrm{a}}$ \\
\hline Reed et al. 1993 [49] & 4 & $34(25-43)$ & $\mathrm{Ib}$ & $\begin{array}{l}1 \mathrm{SH}+\mathrm{CT} \\
3 \mathrm{RH}+\mathrm{LNE}+\mathrm{CT}\end{array}$ & NED (18-28) \\
\hline Hurteau et al. 1995 [30] & 1 & 22 & $\mathrm{Ib}$ & CRH + LNE 32 weeks gestation & NED (14) \\
\hline Kaku et al. 1997 [12] & 5 & $45(33-54)$ & $\mathrm{Ib}$ & $\begin{array}{l}5 \mathrm{RH}+\mathrm{LNE}+\mathrm{BSO}(1 \mathrm{LN}+)+1 \\
\text { RAD }\end{array}$ & NED (9-169) \\
\hline Stanley-Christian et al. 1997 [14] & 3 & $34(27-41)$ & $\mathrm{Ib}_{1}$ & $\mathrm{RH}+\mathrm{LNE}+\mathrm{BSO}$ & NED (publication date) \\
\hline Lu et al. 1998 [50] & 1 & 47 & $\mathrm{Ib}_{1}$ & $\mathrm{RH}+\mathrm{LNE}$ & NED (9) \\
\hline Bouman et al. 1999 [9] & 2 & $34(29-38)$ & $\mathrm{Ib}$ & $\begin{array}{l}1 \mathrm{RH}+\mathrm{LNE} \\
1 \mathrm{SH}+\mathrm{RAD}\end{array}$ & NED (recovery uneventful) \\
\hline Chang et al. 1999 [47] & 1 & 42 & $\mathrm{Ib}$ & $\mathrm{SH}+\mathrm{RAD}$ & NED (13) \\
\hline Lakhtakia et al. 2000 [51] & 1 & 30 & $\mathrm{Ib}$ & $\mathrm{RH}+\mathrm{LNE}+\mathrm{CT}$ & NED (9) \\
\hline Lellé et al. 2000 [52] & 1 & 45 & I nos & $\mathrm{RH}+\mathrm{LNE}$ & NED (9) \\
\hline Khunamornpong et al. 2001 [11] & 14 & $38(22-49)$ & $\mathrm{Ib}$ & $\begin{array}{l}12 \mathrm{RH}+\mathrm{LNE} \\
2 \mathrm{RH}+\mathrm{LNE}+\mathrm{RAD}(2 \mathrm{LN}+)\end{array}$ & NED (21-144) \\
\hline Reale et al. 2001 [53] & 1 & 69 & I nos & $\mathrm{RH}+\mathrm{LNE}$ & NED (60) \\
\hline Polat et al. 2002 [54] & 1 & 38 & I nos & $\mathrm{RH}+\mathrm{LNE}$ & NED (28) \\
\hline Garcea et al. 2003 [27] & 1 & 29 & $\mathrm{Ib}_{1}$ & $\mathrm{RH}+\mathrm{LNE}+\mathrm{RAD}(\mathrm{LN}+)$ & NED (34) \\
\hline Dede et al. 2004 [26] & 1 & 28 & $\mathrm{Ib}_{1}$ & $\begin{array}{l}\text { After termination of the preg- } \\
\text { nancy at } 8 \text { weeks RH }\end{array}$ & $\begin{array}{l}\text { ROD (42), DOD (“on the fifth } \\
\text { year of first diagnosis") }\end{array}$ \\
\hline Utsugi et al. 2004 [55] & 10 & $45(36-64)$ & $\mathrm{Ib}_{1}$ & $\begin{array}{l}9 \mathrm{RH}+\mathrm{LNE} \\
1 \mathrm{RH}+\mathrm{LNE}+\mathrm{CT}\end{array}$ & NED (36-228) \\
\hline Fadare and Zheng 2005 [16] & 1 & 47 & $\mathrm{Ib}_{1}$ & $\mathrm{RH}+\mathrm{LNE}+\mathrm{BSO}$ & $\operatorname{NED}(4,5)$ \\
\hline Heron et al. 2005 [23] & 1 & 32 & $\mathrm{Ib}_{1}$ & $\begin{array}{l}\text { Delivery } 38 \text { weeks, VGA } \\
\text { (cervical polyp), } 1 \text { month pp: } \\
\text { RH+LNE }\end{array}$ & $\begin{array}{l}\text { ROD (44) (episiotomy scar) } \\
\text { NED (96) }\end{array}$ \\
\hline $\begin{array}{l}\text { Gonzalez-Bosquet et al. } 2009 \\
\text { [56] }\end{array}$ & 1 & 28 & $\mathrm{Ib}$ & $\mathrm{RH}+\mathrm{LNE}$ & NED (18) \\
\hline Korach et al. 2009 [10] & 3 & $39(34-65)$ & $\mathrm{Ib}_{1}$ & $3 \mathrm{RH}+\mathrm{LNE}+\mathrm{BSO}$ & $\begin{array}{l}2 \text { NED (78-180) } \\
1 \text { ROD (24), DOD (“few months } \\
\text { later") }\end{array}$ \\
\hline Lai et al. 2011 [25] & 12 & $42(32-52)$ & $\begin{array}{l}10 \mathrm{Ib}_{1} \\
2 \mathrm{Ia}_{2}\end{array}$ & $\begin{array}{l}9 \mathrm{RH}+\mathrm{LNE}+\mathrm{BSO} \\
2 \mathrm{RH}+\mathrm{LNE}(1 \mathrm{LN}+) \\
1 \mathrm{LNE}+\mathrm{RAD} / \mathrm{CT}\end{array}$ & $\begin{array}{l}11 \text { NED (34-162) } \\
1 \text { ROD (alive } 153 \text { mths) }\end{array}$ \\
\hline Choi et al. 2012 [57] & 2 & $52(48-55)$ & $\mathrm{Ib}_{1}$ & $\begin{array}{l}1 \mathrm{RH} \\
1 \mathrm{RH}+\mathrm{LNE}+\mathrm{BSO}\end{array}$ & NED (13-23) \\
\hline Hagiwara et al. 2013 [28] & 5 & $37(30-41)$ & $\mathrm{Ib}_{1}$ & $\begin{array}{l}4 \mathrm{RH}+\mathrm{LNE} \pm \mathrm{BSO} \\
1 \mathrm{RH}+\mathrm{LNE}+\mathrm{RAD}(1 \mathrm{LN}+)\end{array}$ & NED (42-128) \\
\hline He 2013[31] & 1 & 31 & $\mathrm{Ib}_{1}$ & $\begin{array}{l}\text { Biopsy at } 28 \text { weeks (cervi- } \\
\text { cal papilloma), CRH }+ \text { LNE } \\
\text { (36 weeks) }\end{array}$ & NED (84) \\
\hline Lataifeh et al. 2013 [22] & 8 & $37(29-49)$ & $\mathrm{Ib}_{1}$ & $\begin{array}{l}6 \mathrm{RH}+\mathrm{LNE}+\text { Brachy } \\
1 \mathrm{RH}+\mathrm{LNE}+\mathrm{RAD} / \mathrm{CT}(1 \mathrm{LN}+) \\
1 \mathrm{RH}+\mathrm{LNE}\end{array}$ & NED (18-120) \\
\hline
\end{tabular}


Table 2 (continued)

\begin{tabular}{|c|c|c|c|c|c|}
\hline & $\begin{array}{l}\text { Num- } \\
\text { ber of } \\
\text { patients }\end{array}$ & $\begin{array}{l}\text { Average Age, } \\
\text { year (range) }\end{array}$ & FIGO stage & $\begin{array}{l}\text { Surgery and/or additional treat- } \\
\text { ment }\end{array}$ & Outcome (follow-up, months) \\
\hline Kim et al. 2014 [5] & 8 & $47(34-72)$ & $\mathrm{Ib}_{1}$ & $\begin{array}{l}2 \mathrm{RH}+\mathrm{LNE} \\
1 \mathrm{RH}+\mathrm{LNE}+\mathrm{BSO}+\mathrm{RAD} \\
2 \mathrm{RH}+\mathrm{LNE}+\mathrm{USO} \\
1 \mathrm{LRH}+\mathrm{LNE}+\mathrm{USO}+\mathrm{RAD} \\
1 \mathrm{RH}+\mathrm{LNE}+\mathrm{BSO} \\
1 \mathrm{VH}+\mathrm{RAD}\end{array}$ & NED (9-150) \\
\hline Takeuchi et al. 2014 [58] & 1 & 38 & I nos & RH & NED (publication date) \\
\hline Zhao et al. 2016 [15] & 6 & $36(31-42)$ & $\mathrm{Ib}_{1}$ & $\begin{array}{l}2 \mathrm{RVH}+\mathrm{LNE}+\mathrm{BSO}+\mathrm{AT} \\
2 \mathrm{RVH}+\mathrm{LNE}+\mathrm{USO}+\mathrm{AT} \\
2 \mathrm{RVH}+\mathrm{LNE}+\mathrm{BSO}\end{array}$ & NED (7-57) \\
\hline Zhou et al. 2016 [59] & 4 & $55(47-70)$ & $\mathrm{Ib}_{1}$ & $\begin{array}{l}2 \mathrm{RH}+\mathrm{LNE}+\mathrm{BSO} \\
1 \mathrm{RH}+\mathrm{LNE} \\
1 \mathrm{SH}+\mathrm{RAD}\end{array}$ & NED (49-83) \\
\hline Niu et al. 2017 [60] & 4 & $55(47-70)$ & $\mathrm{Ib}_{1}$ & $\begin{array}{l}1 \mathrm{SH}+\mathrm{RAD} \\
1 \text { amputation of cervix }+\mathrm{LNE} \\
1 \mathrm{RH}+\mathrm{LNE} \\
1 \mathrm{RH}+\mathrm{LNE}+\mathrm{BSO}\end{array}$ & $\begin{array}{l}3 \text { NED (8-34) } \\
1 \text { NED (publication date) }\end{array}$ \\
\hline Guo et al. 2018 [6] & 32 & $42(27-66)$ & $\begin{array}{l}3 \mathrm{Ia}_{1} \\
1 \mathrm{Ia}_{2} \\
28 \mathrm{Ib}_{1}\end{array}$ & $\begin{array}{l}12 \mathrm{RH}+\mathrm{LNE}+\mathrm{BSO} \\
8 \mathrm{LRH}+\mathrm{LNE}+\mathrm{BSO} \\
4 \mathrm{NSLRH}+\mathrm{LNE}+\mathrm{BSO} \\
1 \mathrm{LRH}+\mathrm{BSO} \\
3 \mathrm{LRH}+\mathrm{LNE}+\mathrm{BS} \\
1 \mathrm{RVT}+\mathrm{LNE} \\
1 \mathrm{NSARH}+\mathrm{LNE}+\mathrm{BS} \\
1 \mathrm{LRH}+\mathrm{BS} \\
1 \mathrm{CS}+\mathrm{RH}+\mathrm{LNE}+\mathrm{BS} \\
\text { Including } 9 \text { patients with neo-/ } \\
\text { adjuvant treatment (CT and/ } \\
\text { or RAD) }\end{array}$ & $\begin{array}{l}1 \text { ROD (8), (pelvic, adenocarci- } \\
\text { noma), AWD (37) }\end{array}$ \\
\hline Ju et al. 2018 [24] & 11 & $49(31-64)$ & $\begin{array}{l}10 \mathrm{Ib}_{1} \\
1 \mathrm{Ia}_{2}\end{array}$ & $\begin{array}{l}3 \mathrm{MRH}+\mathrm{LNE}+\mathrm{BSO} \\
2 \mathrm{LRH}+\mathrm{LNE}+\mathrm{BSO} \\
2 \mathrm{LMRH}+\mathrm{BSO} \\
1 \mathrm{RH}+\mathrm{LNE}+\mathrm{BSO}+\mathrm{RAD} / \mathrm{CT} \\
\quad(1 \mathrm{LN}+) \\
1 \mathrm{RH}+\mathrm{LNE}+\mathrm{BSO} \\
1 \mathrm{LRH}+\mathrm{LNE} \\
1 \mathrm{LMRH}\end{array}$ & $\begin{array}{l}7 \text { NED } \\
1 \text { ROD (22)(vaginal stump) } \\
1 \text { ROD (42) (liver) } \\
1 \text { ROD (34) (adnexa) } \\
1 \text { ROD (12) (adnexa), DOD (42) }\end{array}$ \\
\hline Wei et al. 2018 [17] & 6 & $42(31-50)$ & $\mathrm{Ib}_{1}$ & $\begin{array}{l}\mathrm{RH} \\
\mathrm{RH}+\mathrm{LNE} \\
\mathrm{RH}+\mathrm{BSO}+\mathrm{LNE} \\
\mathrm{LRH}+\mathrm{LNE} \\
\mathrm{LRH}+\mathrm{BSO}+\mathrm{LNE} \\
\mathrm{LRH}+\mathrm{BSO}+\mathrm{LNE}+\mathrm{CT} / \mathrm{RAD}\end{array}$ & NED (5-113) \\
\hline Zhang et al. 2020 [61] & 3 & $46(37-58)$ & $\mathrm{Ib}_{1}$ & $\begin{array}{l}2 \mathrm{RH}+\mathrm{LNE}+\mathrm{BSO} \\
1 \mathrm{RH}+\mathrm{LNE}+\mathrm{BSO}+\mathrm{CT}\end{array}$ & NED (56-120) \\
\hline
\end{tabular}


Table 2 (continued)

\begin{tabular}{|c|c|c|c|c|c|}
\hline & $\begin{array}{l}\text { Num- } \\
\text { ber of } \\
\text { patients }\end{array}$ & $\begin{array}{l}\text { Average Age, } \\
\text { year (range) }\end{array}$ & FIGO stage & $\begin{array}{l}\text { Surgery and/or additional treat- } \\
\text { ment }\end{array}$ & Outcome (follow-up, months) \\
\hline \multirow[t]{6}{*}{ Chen et al. 2021 [29] } & \multirow[t]{6}{*}{35} & \multirow[t]{6}{*}{$43(32-68)$} & \multirow{6}{*}{$\begin{array}{l}32 \mathrm{Ib}_{1} \\
3 \mathrm{Ia}_{2}\end{array}$} & $19 \mathrm{RH}+\mathrm{LNE}+\mathrm{BSO}(1 \mathrm{LN}+)$ & \multirow[t]{6}{*}{ NED (5-152) } \\
\hline & & & & $10 \mathrm{LRH}+\mathrm{LNE}+\mathrm{BSO}(1 \mathrm{LN}+)$ & \\
\hline & & & & $3 \mathrm{RH}+\mathrm{LNE}+\mathrm{BS}$ & \\
\hline & & & & $1 \mathrm{LRH}+\mathrm{LNE}+\mathrm{BS}$ & \\
\hline & & & & $1 \mathrm{SH}+\mathrm{LNE}+\mathrm{BS}+\mathrm{RAD} / \mathrm{CT}$ & \\
\hline & & & & $\begin{array}{l}1 \mathrm{SH}+\mathrm{BS}+\mathrm{CT} \\
\text { Including further } 11 \text { patients } \\
\text { with adjuvant treatment (CT } \\
\text { and/or RAD) }\end{array}$ & \\
\hline
\end{tabular}

USO unilateral salpingo-oophorectomy, $V H$ vaginal hysterectomy, $S H$ simple hysterectomy, $L R H$ laparoscopic radical hysterectomy, $R V T$ radical vaginal trachelectomy, $R V H$ radical vaginal hysterectomy, $A T$ adjuvant treatment, $L R H$ laparoscopic radical hysterectomy, $N S L R H$ nervesparing laparoscopic radical hysterectomy, $B S$ bilateral salpingectomy, $L M R H$ laparoscopic modified radical hysterectomy, $C R H$ cesarean radical hysterectomy, $R A D$ radiation. $C T$ chemotherapy, $p p$ post-partum. Brachy brachytherapy, $L N$ lymph node, nos not otherwise specified, $A W D$ alive with disease, $N E D$ no evidence of disease, $R O D$ recurrence of disease, $D O D$ dead of disease

${ }^{a}$ Including all patients of both groups

The case series of Lai et al. [25] reported one case of recurrence after $\mathrm{RH}$, bilateral salpingo-oophorectomy, and LNE. The patient was relapse-free for 153 months.

Dede et al. [26] reported a patient at 8 weeks of gestation who received a cervical punch biopsy revealing a VGA. After termination of the pregnancy, $\mathrm{RH}$ was performed. The tumor recurred in the pelvis 42 months after primary surgery. The patient died because of tumoral complications 5 years after the diagnosis of the disease.

In nine patients, at least one affected lymph node could be detected [5, 11, 12, 22, 25, 27-29], three showed a positive LVI [5, 12, 28], and three was LVI negative [27, 29]. In two patients, LVI was not reported [22, 25].

Four children were born in the "invasive management" group: three by cesarean section combined with RH [6,30, $31]$ and one spontaneously [23].

\section{Discussion}

Stage $\mathrm{Ib}_{1}$ cervical cancer is typically treated with invasive management (RH or primary chemoradiation). Several histologic subtypes have been defined, and the particular subtypes may affect prognosis and thus treatment decisions.

VGA has been described as a separate subtype of adenocarcinoma of the cervix; it is well-differentiated and usually associated with a favorable outcome $[3,32]$. The preoperative selection of young patients is an important issue because of the possibility for fertility-sparing or less-invasive treatment. In the "conservative management" group, 54 patients were treated with conization, simple hysterectomy or trachelectomy without adjuvant therapy (radiation, chemotherapy). One patient had recurrent disease in the cervix 25 months after conization [5]. The margins of excision were uninvolved but were close to the tumor. Analysis of adenocarcinoma in situ indicates that achieving negative margins after surgical excision is associated with a significantly lower rate of residual or recurrent disease [33]. The risk of recurrence was lower for patients who underwent a secondary excisional procedure. Goldstein and Mani [34] reported that the risk of residual disease was reduced when a disease-free margin of $10 \mathrm{~mm}$ was achieved.

VGA is frequently associated with adenocarcinoma in situ (40\%) or cervical intraepithelial neoplasia (30\%) [32]. The selection of appropriate patients for "conservative management" has been hampered by uncertainty regarding the natural history of VGA and associated risk of recurrence along with the potential for multifocal lesions that extend beyond the margin of an otherwise satisfactory conization. To maintain fertility in young patients, a conization with a wide disease-free margin, possibly by performing a second resection, should be the goal.

Other histological factors that should be taken into account are depth of stromal invasion and LVI status. These are prognostic factors for recurrence in early-stage cervical cancer [35] and cannot reliably be assessed in a biopsy specimen alone. Grossly, VGA tumors present as friable or polypoid masses, usually protruding from the endocervical canal and manifesting macroscopically as Ib tumors but often with only superficial or no stromal invasion, similar to Ia tumors. To this end, histological evaluation of the tumor-stroma border is necessary. However, approximately $80 \%$ of VGA tumors are radically treated and thus are very 
often overtreated. Over $95 \%$ of stage I VGA tumors have no or only superficial stromal invasion, and only 3\% are LVI positive [6].

In the present review, one positive lymph node was described in the invasive group in nine patients $[11,12,22$, 24, 25, 27-29], whereby four patients were LVI positive, one was negative; in two cases, LVI had not been determined. Six patients were irradiated postoperatively, and no recurrence occurred. Since lymph node involvement was detected in individual cases with VGA, LNE, e.g., laparoscopic pelvic LNE, remains an option (at least in LVI positive patients) even in the case of uterus preservation.

The patient who died in the "conservative management" group had a VGA diagnosed via conization. However, an external review revealed a VGA with an underlying welldifferentiated adenocarcinoma [8]. Alfsen et al. [36], in studying the reproducibility of histological classification of nonsquamous-cell carcinomas of the uterine cervix, reported agreement between reviewers in only 3 of 15 cases of VGA. The nature of accurate histologic diagnosis of VGA is challenging because of the high rate of pretreatment misdiagnosis [8-10, 37]. A punch biopsy prior to treatment very often yielded an incorrect histological diagnosis. Obviously, it can be difficult to predict the final histopathology via examination of a single biopsy, even if poor prognostic features are not present and the VGA seems to be the only entity [9]. Before definitive conservative management is considered, it is prudent to perform conization to exclude the presence of concomitant tumors and to definitively render the diagnosis of VGA. Moreover, in difficult borderline cases consultation of a second pathologist may be necessary.

In addition to the sometimes difficult histological diagnosis of pure VGA, the question of cell spillage due to manipulation of the exophytically growing primary tumor at the cervix is an additional problem [38]. If VGA is present at the cervix at the time of termination of pregnancy or during childbirth, the probability of tumor dissemination is very high. Tumor disseminations at birth are the main concerns for vaginal delivery through a cervix with cancer [39]. This explains the recurrences in this review [23, 26]. If the VGA had been removed via conization before the termination of pregnancy or before birth, a relapse would most likely not have occurred.

The three cases of intraabdominal metastases after minimally invasive surgery in the paper by Ju et al.[24] are probably also related to this problem. These three patients had no risk factors for metastasis (no LVI and no lymph node involvement and had superficial invasion only). In all 15 cases, VGA was diagnosed after a punch biopsy. Among potential reasons for the inferior oncological outcomes in patients with cervical cancer who underwent minimally invasive surgery than in women who underwent open surgery, the routine use of a uterine manipulator might increase the propensity for tumor spillage intraperitoneally after colpotomy under laparoscopic vision [40, 41].

The present literature review provides some evidence that the manipulation ("excessive handling") of cervical VGA can worsen the prognosis of this tumor. Of the 11 cases of recurrence, the vast majority could most likely have been avoided if, first, the VGA at the cervix had been preventively removed by conization with tumor-free margins and, second, the exact histological diagnosis had been made by a qualified gynecopathologist.

The strengths of this study include the largest systematic review 1989-2021 of this rare tumor and the first attempt to compare a non-radical (conservative) with a radical (invasive) approach. However, our conclusions were limited by the retrospective view of the data and the number of VGA tumors was limited for this rare tumor. Thus, it is suggested to perform multicenter prospective studies to investigate diagnosis and optimal treatment of this subtype of cervical cancer.

The DFS of the conservative group is comparable to the invasive group $(p=0.75)$. Radical surgery in the invasive group does not lead to better results compared to the conservative group. Since these VGA tumors can always be visualized on gynecologic examination due to their exophytic growth and are accordingly classified as stage Ib (FIGO), most patients in the invasive group were treated with radical hysterectomy, as standard therapy for cervical cancer, although conization with wide negative margins would most likely have been sufficient for diagnosis and therapy. It would still have been possible to modify the therapy after conization depending on the stromal infiltration in the sense of a "patient-tailored surgical treatment". In addition, conization can improve the prognosis of common cervical carcinoma [42].

Histopathological evidence of VGA should be included in the treatment decision and prognosis estimation in the multidisciplinary tumor conference. It is essential that VGA is treated as a special subtype of cervical carcinoma with an excellent prognosis. Awareness of this special form and decision-making strictly based on the histology of the conisate regarding possible further conservative or invasive therapy should be present.

In conclusion, VGA is a complex tumor that has an excellent prognosis in its pure histological appearance. It is not justified to lump VGA and usual cervical cancer together and to perform radical surgery. In any case, the decisive step towards adequate treatment for VGA is a qualified histological diagnosis that excludes a less differentiated carcinoma component. A pretherapeutic conization with wide tumor-free margins is an indispensable prerequisite for this decision. We believe that patients could benefit from this low-risk histology and the next step could be only a sentinel 
node mapping [43]. In pure VGA, conservative management is justifiable, especially for young women, and a radical approach may result in overtreatment.

Author contribution AD: data collection, data analysis, and manuscript writing. KA, MWB: manuscript writing.

Funding Open Access funding enabled and organized by Projekt DEAL.

\section{Declarations}

Conflict of interest We declare that we have no conflict of interest.

Availability of data and material The datasets used and/or analyzed during for the presented manuscript are available from the corresponding author on reasonable request.

Open Access This article is licensed under a Creative Commons Attribution 4.0 International License, which permits use, sharing, adaptation, distribution and reproduction in any medium or format, as long as you give appropriate credit to the original author(s) and the source, provide a link to the Creative Commons licence, and indicate if changes were made. The images or other third party material in this article are included in the article's Creative Commons licence, unless indicated otherwise in a credit line to the material. If material is not included in the article's Creative Commons licence and your intended use is not permitted by statutory regulation or exceeds the permitted use, you will need to obtain permission directly from the copyright holder. To view a copy of this licence, visit http://creativecommons.org/licenses/by/4.0/.

\section{References}

1. Smith HO, Tiffany MF, Qualls CR, Key CR (2000) The rising incidence of adenocarcinoma relative to squamous cell carcinoma of the uterine cervix in the United States-a 24-year populationbased study. Gynecol Oncol 78(2):97-105. https://doi.org/10. 1006/gyno.2000.5826

2. Bulk S, Visser O, Rozendaal L, Verheijen RH, Meijer CJ (2005) Cervical cancer in the Netherlands 1989-1998: decrease of squamous cell carcinoma in older women, increase of adenocarcinoma in younger women. Int J Cancer 113(6):1005-1009. https://doi. org/10.1002/ijc. 20678

3. Young RH, Scully RE (1989) Villoglandular papillary adenocarcinoma of the uterine cervix. A clinicopathologic analysis of 13 cases. Cancer 63(9):1773-1779. https://doi.org/10.1002/10970142(19900501)63:9\%3c1773::aid-cncr2820630920\%3e3.0.co;2-j

4. Hodgson A, Olkhov-Mitsel E, Howitt BE, Nucci MR, Parra-Herran C (2019) International Endocervical Adenocarcinoma Criteria and Classification (IECC): correlation with adverse clinicopathological features and patient outcome. J Clin Pathol 72(5):347-353. https://doi.org/10.1136/jclinpath-2018-205632

5. Kim HJ, Sung JH, Lee E, Ahn S, Song SY, Choi CH, Kim TJ, Kim BG, Bae DS, Lee JW (2014) Prognostic factors influencing decisions about surgical treatment of villoglandular adenocarcinoma of the uterine cervix. Int J Gynecol Cancer 24(7):1299-1305. https://doi.org/10.1097/igc.0000000000000197

6. Guo P, Liu P, Yang J, Ren T, Xiang Y (2018) Villoglandular adenocarcinoma of cervix: pathologic features, clinical management, and outcome. Cancer Manag Res 10:3955-3961. https://doi.org/ 10.2147/cmar.S165817

7. Moher D, Liberati A, Tetzlaff J, Altman DG (2009) Preferred reporting items for systematic reviews and meta-analyses: the PRISMA statement. BMJ 339:b2535. https://doi.org/10.1136/ bmj.b2535

8. Macdonald RD, Kirwan J, Hayat K, Herrington CS, Shawki H (2006) Villoglandular adenocarcinoma of the cervix: clarity is needed on the histological definition for this difficult diagnosis. Gynecol Oncol 100(1):192-194. https://doi.org/10.1016/j.ygyno. 2005.07.133

9. Bouman A, Oosterhuis GJ, Naudin ten Cate L, van Doorn GA (1999) Villoglandular papillary adenocarcinoma of the cervix. Beware of a wolf in sheep's clothing. Eur J Obstet Gynecol Reprod Biol. 87(2):183-189. https://doi.org/10.1016/s03012115(99)00106-2

10. Korach J, Machtinger R, Perri T, Vicus D, Segal J, Fridman E, Ben-Baruch G (2009) Villoglandular papillary adenocarcinoma of the uterine cervix: a diagnostic challenge. Acta Obstet Gynecol Scand 88(3):355-358. https://doi.org/10.1080/000163409027303 59

11. Khunamornpong S, Maleemonkol S, Siriaunkgul S, Pantusart A (2001) Well-Differentiated villoglandular adenocarcinoma of the uterine cervix: a report of 15 cases including two with lymph node metastasis. J Med Assoc Thai 84(6):882-888

12. Kaku T, Kamura T, Shigematsu T, Sakai K, Nakanami N, Uehira K, Amada S, Kobayashi H, Saito T, Nakano H (1997) Adenocarcinoma of the uterine cervix with predominantly villogladular papillary growth pattern. Gynecol Oncol 64(1):147-152. https:// doi.org/10.1006/gyno.1996.4539

13. Hopson L, Jones MA, Boyce CR, Tarraza HM Jr (1990) Papillary villoglandular carcinoma of the cervix. Gynecol Oncol 39(2):221224. https://doi.org/10.1016/0090-8258(90)90437-p

14. Stanley-Christian H, Heim BK, Hines JF, Hall KL, Willett GD, Barnes WA (1997) Villoglandular adenocarcinoma of the cervix: a report of three cases and review of the literature. Gynecol Oncol 66(2):327-330. https://doi.org/10.1006/gyno.1997.4747

15. Zhao L, Xu T, Cui M, Fu Z (2016) A retrospective review of 11 cases of villoglandular papillary adenocarcinoma of the uterine cervix and a review of the literature. Oncol Lett 11(3):2164-2168. https://doi.org/10.3892/ol.2016.4172

16. Fadare O, Zheng W (2005) Well-differentiated papillary villoglandular adenocarcinoma of the uterine cervix with a focal highgrade component: is there a need for reassessment? Virchows Arch 447(5):883-887. https://doi.org/10.1007/s00428-005-0030-3

17. Wei C-Y, Qu Y-Q, He Y-Y, Wang Q, Zhu X-Y, Shao J (2018) A retrospective review of 10 cases of villoglandular papillary adenocarcinoma of the uterine cervix including one with successful pregnancy. Reprod Dev Med 2(2):120-127

18. Lavie O, Segev Y, Peer G, Gutterman E, Sagie S, Auslnader R (2008) Conservative management for villoglandular papillary adenocarcinoma of the cervix diagnosed during pregnancy followed by a successful term delivery: a case report and a review of the literature. Eur J Surg Oncol 34(5):606-608. https://doi.org/ 10.1016/j.ejso.2007.05.014

19. Takai N, Hayashita C, Nakamura S, Narahara H, Matsumoto H (2010) A case of villoglandular papillary adenocarcinoma of the uterine cervix diagnosed during early pregnancy followed by successful term delivery. Case Rep Med 2010:314547. https://doi.org/ $10.1155 / 2010 / 314547$

20. Hoffman JS, Bazzurini L, Laird L, Murphy JC, Magriples U, Lewis J (2001) Term delivery following conservative treatment for villoglandular papillary adenocarcinoma of the uterine cervix: report of a case and analysis of the literature. Gynecol Oncol 81(2):310-313. https://doi.org/10.1006/gyno.2001.6129 
21. Falcón O, García R, Lubrano A, Morín JC, Andujar M (2006) Successful term delivery following conservative management for villoglandular papillary adenocarcinoma of the uterine cervix: a case report. Gynecol Oncol 101(1):168-171. https://doi.org/10. 1016/j.ygyno.2005.09.059

22. Lataifeh IM, Al-Hussaini M, Uzan C, Jaradat I, Duvillard P, Morice P (2013) Villoglandular papillary adenocarcinoma of the cervix: a series of 28 cases including two with lymph node metastasis. Int J Gynecol Cancer 23(5):900-905. https://doi.org/ 10.1097/IGC.0b013e31828efcaa

23. Heron DE, Axtel A, Gerszten K, Amortegui A, Kelley J, Comerci J, Edwards RP (2005) Villoglandular adenocarcinoma of the cervix recurrent in an episiotomy scar: a case report in a 32-year-old female. Int J Gynecol Cancer 15(2):366-371. https://doi.org/10. 1111/j.1525-1438.2005.15231.x

24. Ju UC, Kang WD, Kim SM (2018) Is the ovarian preservation safe in young women with stages IB-IIA villoglandular adenocarcinoma of the uterine cervix? J Gynecol Oncol 29(4):e54. https:// doi.org/10.3802/jgo.2018.29.e54

25. Lai J-Y, Chen J-R, Chen Y-J, Hsu C-H, Wang T-Y, Yang Y-C (2011) Villoglandular adenocarcinoma of the uterine cervix: an analysis of 12 clinical cases. Int J Gerontol 5(1):49-52

26. Dede M, Deveci G, Deveci MS, Yenen MC, Goktolga U, Dilek S, Gunhan O (2004) Villoglandular papillary adenocarcinoma of the uterine cervix in a pregnant woman: a case report and review of literature. Tohoku J Exp Med 202(4):305-310. https://doi.org/ 10.1620/tjem.202.305

27. Garcea A, Nunns D, Ireland D, Brown L (2003) A case of villoglandular papillary adenocarcinoma of the cervix with lymph node metastasis. BJOG 110(6):627-629

28. Hagiwara T, Kaku T, Kobayashi H, Wake N, Saito T (2013) Welldifferentiated villoglandular adenocarcinoma of the uterine cervix: assessment of cytological features by histological subtypes. Acta Cytol 57(1):61-68. https://doi.org/10.1159/000342917

29. Chen JH, Duan H, Yu XB, Zhao HW, Chen X, Li P, Li ZQ, Li BX, Pan LY, Yan X, Chen C (2021) Clinical features and prognostic factors of cervical villoglandular adenocarcinoma. Int J Gynecol Cancer 31(4):512-517. https://doi.org/10.1136/ijgc-2020-002044

30. Hurteau JA, Rodriguez GC, Kay HH, Bentley RC, Clarke-Pearson D (1995) Villoglandular adenocarcinoma of the cervix: a case report. Obstet Gynecol 85(5 Pt 2):906-908. https://doi.org/10. 1016/0029-7844(94)00418-d

31. He C (2013) Villoglandular papillary adenocarcinoma of the uterine cervix diagnosed during pregnancy: a case report. Gynecology \& Obstetrics 3(3):155

32. Jones MW, Silverberg SG, Kurman RJ (1993) Well-differentiated villoglandular adenocarcinoma of the uterine cervix: a clinicopathological study of 24 cases. Int J Gynecol Pathol 12(1):1-7. https://doi.org/10.1097/00004347-199301000-00001

33. Salani R, Puri I, Bristow RE (2009) Adenocarcinoma in situ of the uterine cervix: a metaanalysis of 1278 patients evaluating the predictive value of conization margin status. Am J Obstet Gynecol 200(2):182.e181-185. https://doi.org/10.1016/j.ajog.2008.09.012

34. Goldstein NS, Mani A (1998) The status and distance of cone biopsy margins as a predictor of excision adequacy for endocervical adenocarcinoma in situ. Am J Clin Pathol 109(6):727-732. https://doi.org/10.1093/ajcp/109.6.727

35. Bentivegna E, Gouy S, Maulard A, Chargari C, Leary A, Morice $P$ (2016) Oncological outcomes after fertility-sparing surgery for cervical cancer: a systematic review. Lancet Oncol 17(6):e240 e253. https://doi.org/10.1016/s1470-2045(16)30032-8

36. Alfsen GC, Reed W, Abeler VM (2003) Reproducibility of classification in non-squamous cell carcinomas of the uterine cervix. Gynecol Oncol 90(2):282-289. https://doi.org/10.1016/s00908258(03)00280-4
37. Heatley MK (2007) Villoglandular adenocarcinoma of the uterine cervix-a systematic review of the literature. Histopathology 51(2):268-269. https://doi.org/10.1111/j.1365-2559.2007.02759.x

38. Dietl A, Aumann K, Beckmann MW (2020) Tumor handling of early-stage cervical cancer: a literature analysis of villoglandular adenocarcinoma of the cervix. Anticancer Res 40(6):3049-3053. https://doi.org/10.21873/anticanres.14285

39. La Russa M, Jeyarajah AR (2016) Invasive cervical cancer in pregnancy. Best Pract Res Clin Obstet Gynaecol 33:44-57. https:// doi.org/10.1016/j.bpobgyn.2015.10.002

40. Dietl A, Klar M, Aumann K (2019) Minimally invasive surgery for early-stage cervical cancer: is the uterine manipulator a risk factor? Am J Obstet Gynecol 221(5):537-538. https://doi.org/10. 1016/j.ajog.2019.07.042

41. Fader AN (2018) Surgery in cervical cancer. N Engl J Med 379(20):1955-1957. https://doi.org/10.1056/NEJMe1814034

42. Benoit L, Koual M, Nguyen-Xuan HT, Balaya V, Nos C, MonteroMacías R, Bats AS (2021) Does a pre-operative conization improve disease-free survival in early-stage cervical cancer? Arch Gynecol Obstet 303(1):231-239. https://doi.org/10.1007/ s00404-020-05798-7

43. Dabi Y, Willecocq C, Ballester M, Carcopino X, Bendifallah S, Ouldamer L, Lavoue V, Canlorbe G, Raimond E, Coutant C, Graesslin O, Collinet P, Bricou A, Huchon C, Daraï E, Haddad B, Touboul C (2018) Identification of a low risk population for parametrial invasion in patients with early-stage cervical cancer. J Transl Med 16(1):163. https://doi.org/10.1186/ s12967-018-1531-6

44. Skopelitou A, Hadjiyannakis M (1996) Enteric type villoglandular papillary adenocarcinoma of the uterine cervix associated with in situ squamous cell carcinoma. Case report and review of the literature. Eur J Gynaecol Oncol 17(4):309-314

45. Novotny DB, Ferlisi P (1997) Villoglandular adenocarcinoma of the cervix: cytologic presentation. Diagn Cytopathol 17(5):383387. https://doi.org/10.1002/(sici)1097-0339(199711)17:5\% 3c383::aid-dc13\%3e3.0.co;2-j

46. Borgo G, Feyles E, Gaglio A, Tagliani L, Andrion A (1998) Villoglandular papillary adenocarcinoma of the uterine cervix: report of a case. Tumori 84(6):717-719

47. Chang WC, Matisic JP, Zhou C, Thomson T, Clement PB, Hayes MM (1999) Cytologic features of villoglandular adenocarcinoma of the uterine cervix: comparison with typical endocervical adenocarcinoma with a villoglandular component and papillary serous carcinoma. Cancer 87(1):5-11. https://doi.org/10. 1002/(sici)1097-0142(19990225)87:1\%3c5::aid-cncr2\%3e3.0. co;2-d

48. Dilley S, Newbill C, Pejovic T, Munro E (2015) Two cases of endocervical villoglandular adenocarcinoma: support for conservative management. Gynecol Oncol Rep 12:34-36. https://doi.org/ 10.1016/j.gore.2015.02.004

49. Reed W, Abeler VM, Tropé CG (1993) Villous glandular adenocarcinoma of the uterine cervix. A subtype with favourable prognosis. Tidsskrift Nor Laegeforening 113(20):2569-2571

50. Lu FH, Chen BF, Yang YC (1998) Well-differentiated papillary villoglandular adenocarcinoma of the uterine cervix: a case report. Zhonghua Yi Xue Za Zhi (Taipei) 61(7):436-440

51. Lakhtakia R, Singh MK, Taneja P, Kapila K, Kumar S (2000) Villoglandular papillary adenocarcinoma of the cervix: case report. J Surg Oncol 74(4):297-299. https://doi.org/10.1002/10969098(200008)74:4\%3c297::aid-jso12\%3e3.0.co;2-3

52. Lellé R, Maier E, Eltze E, Böcker W (2000) Villoglandular carcinoma: a rare manifestation of cervical adenocarcinoma with good prognosis. Case report and review of the literature. Geburtshilfe Frauenheilkd 60(8):436-439 
53. Reale D, Vitullo G, Di Virgilio M, Trubiani O, Pizzicannella G (2001) Villograndular adenocarcinoma of uterine cervix: a case report. Pathologica 93(2):128-131

54. Polat A, Düsmez D, Pata O, Aydin O, Egilmez R (2002) Villoglandular papillary adenocarcinoma of the uterine cervix with immunohistochemical characteristics. J Exp Clin Cancer Res 21(3):425-427

55. Utsugi K, Shimizu Y, Akiyama F, Umezawa S, Hasumi K (2004) Clinicopathologic features of villoglandular papillary adenocarcinoma of the uterine cervix. Gynecol Oncol 92(1):64-70. https:// doi.org/10.1016/j.ygyno.2003.10.020

56. González-Bosquet E, Suñol M, Morante D, Gomez Latre M, Callejo J, Lailla JM (2009) Villoglandular papillary adenocarcinoma of the uterine cervix: a case report and literature review. Eur J Gynaecol Oncol 30(2):211-213

57. Choi Y, Kim H, Choi H, Hwang D, Choe G, Chung JH, Park SY, Lee HS, Paik JH, Park HJ (2012) Liquid-based cytology of villoglandular adenocarcinoma of the cervix: a report of 3 cases. Korean J Pathol 46(2):215-220. https://doi.org/10.4132/KoreanJPathol. 2012.46.2.215

58. Takeuchi M, Matsuzaki K, Bando Y, Sakaki M, Furumoto H, Harada M (2014) Magnetic resonance manifestations of villoglandular papillary adenocarcinoma of the uterine cervix with a fern-leaf-like appearance. Magn Reson Med Sci 13(4):267-270. https://doi.org/10.2463/mrms.2013-0078

59. Zhou QY, Chen HY, Yang SM, Li YH, Wu XQ (2016) Villoglandular papillary adenocarcinoma of the uterine cervix: A report of 4 cases and a review of the literature. Oncol Lett 11(1):837-841. https://doi.org/10.3892/ol.2015.3944

60. Niu Q, Guan J, Zhang Y, Xi R, Zhang H (2017) Four cases of villoglandular papillary adenocarcinoma and literature review. Biomedical Research. S443-S446

61. Zhang Y, Wang Y, Liu Y, Yang J, Liu C (2020) A retrospective study and literature review of cervical villoglandular adenocarcinoma: a candidate paradigm of silva system pattern A. Appl Immunohistochem Mol Morphol. https://doi.org/10.1097/pai. 0000000000000895

Publisher's Note Springer Nature remains neutral with regard to jurisdictional claims in published maps and institutional affiliations. 